\title{
Assessment of variability in traction interventions for patients with low back pain: a systematic review
}

\author{
Muhammad Alrwaily ${ }^{1,2^{*}}$, Mohammed Almutiri ${ }^{3}$ and Michael Schneider ${ }^{4}$
}

\begin{abstract}
Background: Previous systematic reviews have concluded that lumbar traction is not effective for patients with low back pain (LBP), yet many clinicians continue to assert its clinical effectiveness.

Objective: To systematically identify randomized controlled trials (RCTs) of traction and explore the variability of traction interventions used in each RCT.

Method: A literature search started in September 2016 to retrieve systematic reviews and individual RCTs of lumbar traction. The term "lumbar traction" and other key words were used in the following databases: Cochrane Registry, MEDLINE, EMBASE, and CINAHL. The retrieved systematic reviews were used to extract individual RCTs. The most current systematic review included RCTs from inception until August 2012. We performed an additional literature search to update this systematic review with newer RCTs published between September 2012 and December 2016. All of the identified RCTs were combined and summarized into a single evidence table.

Results: We identified a total of 37 traction RCTs that varied greatly in their method of traction intervention. The RCTs included several types of traction: mechanical (57\%), auto-traction (16\%), manual (10.8\%), gravitational (8.1\%) and aquatic (5.4\%). There was also great variability in the types of traction force, rhythm, session duration and treatment frequency used in the RCTs. Patient characteristics were a mixture of acute, subacute and chronic LBP; with or without sciatica.

Conclusion: There is wide variability in the type of traction, traction parameters and patient characteristics found among the RCTs of lumbar traction. The variability may call into question the conclusion that lumbar traction has little no or value on clinical outcomes. Also, this variability emphasizes the need for targeted delivery methods of traction that match appropriate dosages with specific subgroups of patients with LBP.
\end{abstract}

Keywords: Traction, Low back pain, Sciatica, Systematic review

\section{Introduction}

Lumbar traction is a commonly used method to treat patients with low back pain (LBP) with or without sciatica. In the UK and the US, lumbar traction is used by 41 and $77 \%$ of outpatient rehabilitation providers respectively $[1,2]$. Despite this common use of lumbar traction in the clinical setting, several systematic reviews have concluded that lumbar traction has little or no value on the clinical

\footnotetext{
* Correspondence: muhammad.alrwaily@hsc.wvu.edu

'Division of Physical Therapy, School of Medicine, West Virginia University, 1

Medical Center Drive, P.O. Box 9226 - Room 8304, Morgantown, WV 26506,

USA

${ }^{2}$ King Fahad Specialist Hosptial, Dammam, Saudi Arabia

Full list of author information is available at the end of the article
}

outcomes of pain intensity and functional status. The reviews also suggest that traction does not appear to lead to quicker return to work among people with LBP with or without sciatica [3-5]. These conclusions present a clear discordance between evidence-based recommendations and how lumbar traction is regarded in current clinical practice $[1,2,6]$.

The earliest systematic review, conducted in 1995, included 17 randomized controlled trials (RCTs) that assessed traction on neck and low back pain [7]. Of the 17 RCTs, only 3 (2 lumbar, 1 cervical) had good quality. This systematic review concluded that traction efficacy

(c) The Author(s). 2018 Open Access This article is distributed under the terms of the Creative Commons Attribution 4.0 International License (http://creativecommons.org/licenses/by/4.0/), which permits unrestricted use, distribution, and reproduction in any medium, provided you give appropriate credit to the original author(s) and the source, provide a link to the Creative Commons license, and indicate if changes were made. The Creative Commons Public Domain Dedication waiver (http://creativecommons.org/publicdomain/zero/1.0/) applies to the data made available in this article, unless otherwise stated. 
was unclear, and called for more proper design and better methodological quality in future traction trials.

An update of the above systematic review, published in 2006, included 24 RCTs that assessed the effectiveness of traction in the management of LBP [4]. The RCTs were selected if they examined any type of traction on acute, subacute, or chronic LBP with or without sciatica. Of the 24 RCTs, only 5 were considered of high quality, and suggested that there was strong evidence that traction was not effective in the management of patients with mixed duration of LBP with or without sciatica. However, there was moderate evidence that autotraction was effective in the management of patients with mixed duration of LBP with or without sciatica.

The most recent update of the above systematic reviews, published in 2013, included 32 RCTs that assessed the effectiveness of traction in management of LBP using the same selection criteria that were used previously [5]. Of the 32 RCTs, 16 studies were considered to have low-risk of bias. The overall conclusion of this systematic review suggested that traction, alone or in combination with other interventions, has little or no impact on the clinical outcomes of pain and function on people with mixed duration of LBP with or without sciatica. However, this systematic review suggested that large, high-quality studies, were still required to make definitive conclusion about traction effectiveness.

Interestingly, rehabilitation providers are reported to be aware of the recommendations from systematic reviews against traction, yet $64 \%$ of them disagree with these recommendations and $25 \%$ remain undecided [1]. One explanation for this large amount of disagreement may be that rehabilitation providers regularly report the empirical observation that some patients are dramatic responders to lumbar traction [1]. This clinical observation may be driven by the ability of rehabilitation providers to somehow recognize certain clinical patterns that allow them to match patients' symptomatic presentations to specific traction strategies [2]. This pattern recognition of a traction subgroup has been recommended within the treatment-based classification system, which is commonly utilized by physical therapists [8-10].

Another explanation for this divergence between the continued use of lumbar traction by rehabilitation providers and the recommendations against it from systematic reviews may be related to the variability in the delivery methods of traction among the RCTs included in these systematic reviews [5]. The variability in delivery of traction interventions can stem from using different types of traction, different traction parameters, and different patient populations [5]. When RCTs with different traction methods are pooled together, the overall treatment effect size is diluted.
A number of RCTs suggest that traction can be an effective intervention in the management of patients with LBP. Fritz et al. found that mechanical traction in combination with extension exercises can result in significant improvement in disability and fear-avoidance beliefs after two weeks of treatment compared to extension exercises alone for patients with acute LBP and nerve root compression symptoms [11]. Also, Prasad et al. found that using inversion traction plus physical therapy in patients awaiting surgery for disc herniation helped $77 \%$ of them avoid surgery compared to physical therapy alone that helped only $22 \%$ avoid surgery [12]. Additionally, Kim et al. found that when prescribing the inversion traction for patients with chronic LBP, the tilt degree of the traction table matters [13]. Kim et al. found the a tilt degree of 60 resulted in improve levels of pain, spine flexibility and trunk extensors strength compared to tilt degrees of 30 or 0 (supine position) [13]. Further, Simmerman et al. found that aquatic traction resulted in significant pain reduction and centralization of symptoms compared to land-based exercises in patients with chronic LBP associated with nerve root compression symptoms [14]. Finally, Diab and Moustafa found that traction in combination with stretching and infrared radiation resulted in significant improvement of pain and disability levels compared to stretching and infrared in patients with chronic LBP at 6 months [15]. Collectively, these individual RCTs point to the potential effectiveness of traction in the treatment of patients with LBP with or without sciatica.

Given the possibility that the treatment effect size could be diluted when heterogeneous studies are pooled together, the purpose of this review is to map the evidence regarding the diversity in traction delivery methods. Specifically, this systematic review will explore the various traction intervention protocols by reporting on traction types, traction parameters, dosage and patients' characteristics. Assessing the diversity of traction delivery methods will help determine the appropriateness of conducting meta-analysis.

\section{Methods}

This is a systematic review of RCTs that have included some type of lumbar traction as a treatment intervention. The data collection of this systemic review started in September 2016. The first step was to perform a generalized search of the literature using the key words "lumbar traction" in the following databases: Cochrane Central Register of Controlled Trials, MEDLINE, EMBASE, and CINAHL. The search returned several systematic reviews that directly addressed the topic of lumbar traction. The most recent Cochrane systematic review included RCTs of lumbar traction published from inception until August 2012 [5]. From this Cochrane 
review [5], all of the RCTs contained within the evidence tables were extracted and compared with those found in the evidence tables of the other systematic reviews $[3,7]$. This step was necessary to confirm that the recent Cochrane review included all of the RCTs (or more) contained in the evidence tables of the older systematic reviews.

The next step was to update the systematic review by searching for additional RCTs published between September 2012 and December 2016. To identify new RCTs, the following keywords were used to search the same databases: "traction" OR "traction therapy" OR "traction physical therapy" OR "decompression" OR "unloading"; OR "lower back" OR "low back pain" OR lumbar pain OR sciatica OR radiculopathy OR lumbago OR backache. After these additional RCTs were retrieved, two authors (MAlr and MAlm) examined the titles and abstracts to select studies that would potentially be worthy of full text review. After that, the two authors extracted and synthesized the data about the specific traction protocols used in each RCT by reporting on the traction type, traction parameters and patient population. The authors used consensus to agree on which trials would warrant a review of the full text article for potential inclusion in this systematic review. When disagreement occurred, the third author (MS) was consulted to resolve the disagreement.

For a study to be included in this systematic review, it had to be an RCT of patients 18 years of age or older; with acute, subacute or chronic LBP; with or without sciatica. Also, the studies had to include at least one type of traction: manual, auto-traction, gravitational, aquatic and mechanical traction. The traction may or may not have been combined with other interventions such as manual therapy or exercise, with the requirement that traction was the primary intervention. Additionally, any type of comparison group was allowed including placebo, sham or active intervention. Finally, the RCTs must have included at least one clinically relevant outcome measure such as numeric pain scale, self-reported function, global measure of improvement, or return to work. These inclusion criteria are similar to those reported in the most recent Cochrane systematic review [5].

The last step was to create an evidence table that combined the RCTs extracted from the most recent Cochrane systematic review with the new RCTs identified through the updated search (Table 1). Because this review focused on extracting details about the specific traction protocols used in each RCT, there was no need to collect data on quality and risk of bias. This format was used to allow the reader to quickly visualize the similarities and differences in traction protocols across the RCTs.

\section{Results}

The most recent Cochrane systematic review included 32 individual lumbar traction RCTs published from inception through August 2012 [3, 5, 7]. Our updated search identified an additional 14 newer studies that were published between September 2012 and December 2016. Of these newer studies, 5 RCTs were combined in Table 1 with the previously identified 32 RCTs for a grand total of 37 RCTs [12, 13, 15-17]. This search process is summarized in (Fig. 1) [18].

In the columns of Table 1, the primary author and date of each RCT are organized in chronological order. In the rows of Table 1 , the qualitative factors and traction parameters of each RCT were included. One study included the results of two RCTs in a single publication [19], so both of those two RCTs were included in Table 1, each reported in a separate column.

Table 2 uses descriptive statistics to summarize categories of traction parameters and patient characteristics from the included RCTs. Of the 37 RCTs, $59.5 \%$ used some type of mechanical traction while the remaining studies used auto-traction (16.2\%), manual (10.8\%), inversion $(8.1 \%)$, or aquatic traction (5.4\%). In $27 \%$ of the trials, traction was used in combination with some other type of rehabilitation intervention, such as exercise or physical agents.

The amount of force used during the traction treatment varied widely across these 37 studies, ranging from $2.3 \mathrm{~kg}$ in one trial to $100 \%$ of body weight in another. In $35 \%$ of the RCTs, the amount of traction force was determined by using some arbitrary percentage of the patient's body weight that varied from 20 to $100 \%$. However, another $37.8 \%$ of studies used an arbitrary pre-determined amount of weight ranging anywhere from $2.3 \mathrm{~kg}$ to $60 \mathrm{~kg}$ as the traction force. The traction rhythm was evenly distributed between continuous and intermittent types of application, with each type of application used $43.2 \%$ of the time. In the remaining of the studies, the traction force and rhythm were not clearly described.

The traction session time and treatment frequencies were very difficult to categorize. The traction sessions lasted from 3 to $4 \mathrm{~min}$ in duration in some trials, to more than $30 \mathrm{~min}$ in other trials. The frequency of application of the traction treatments used in these trials varied from as few as one single session, to as many as 20 traction sessions applied over 6 to 10 weeks.

Other traction parameters were also found to vary widely across these 37 traction RCTs. With respect to patient positioning during the application of traction, $29.7 \%$ of the trials had the patient lie in a supine position, $16.2 \%$ used prone positioning, 5\% applied traction in standing, 3\% used a side-lying position, and in $43.2 \%$ of the studies there was no clear description of patient positioning. 


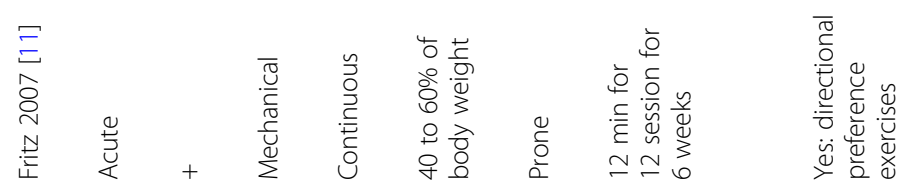

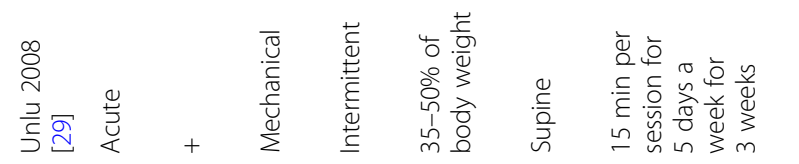

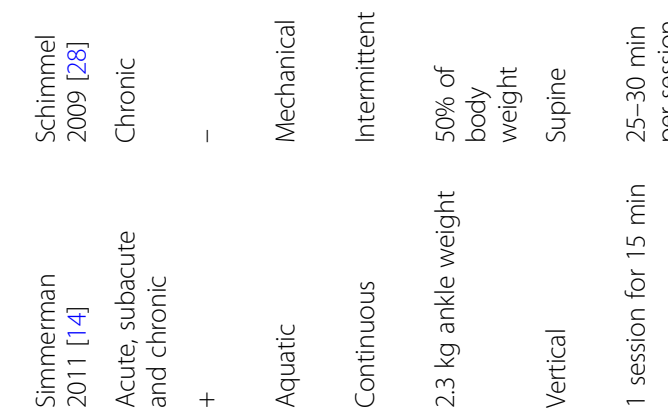

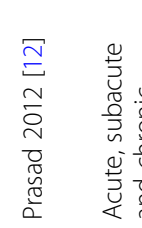

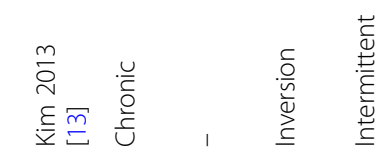

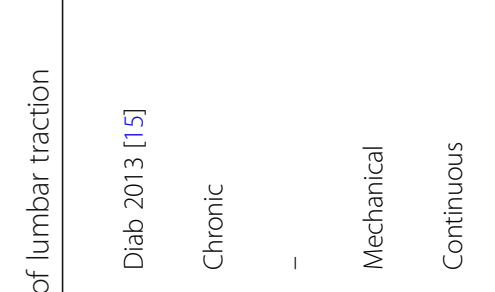

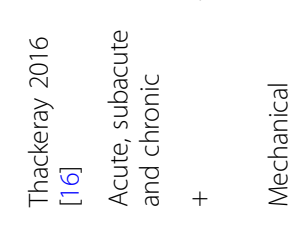

苍

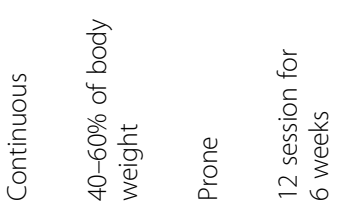

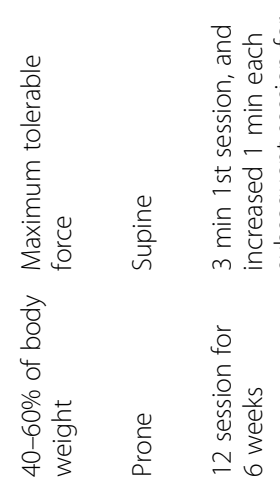

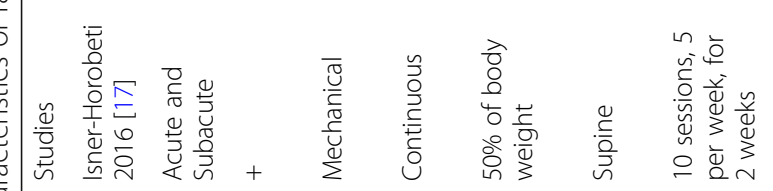

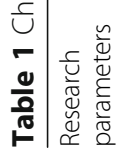

焦

之

z

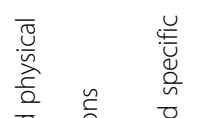

离

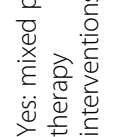

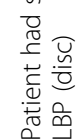

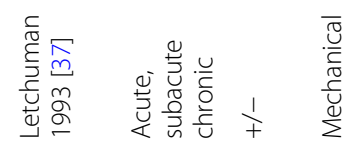

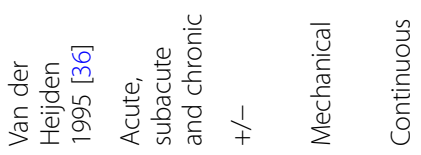

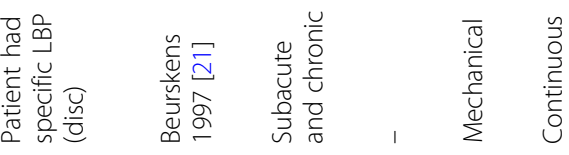

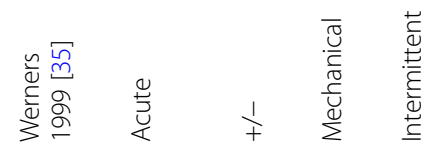

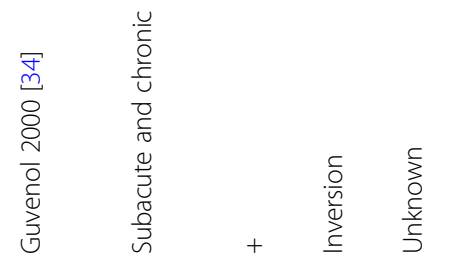

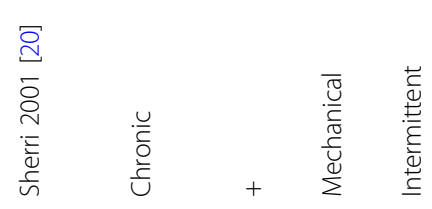

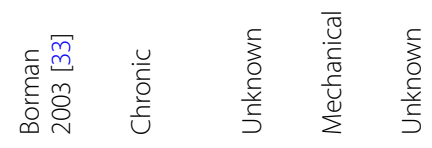

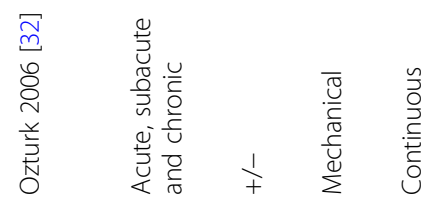

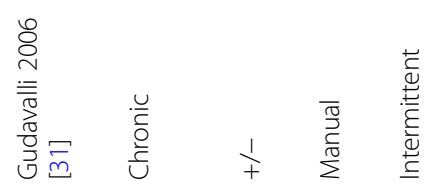

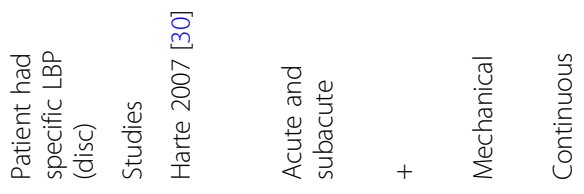

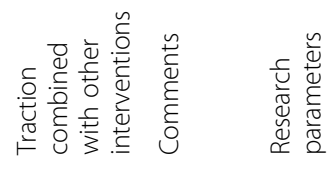

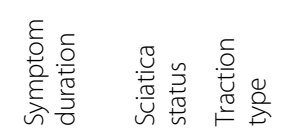




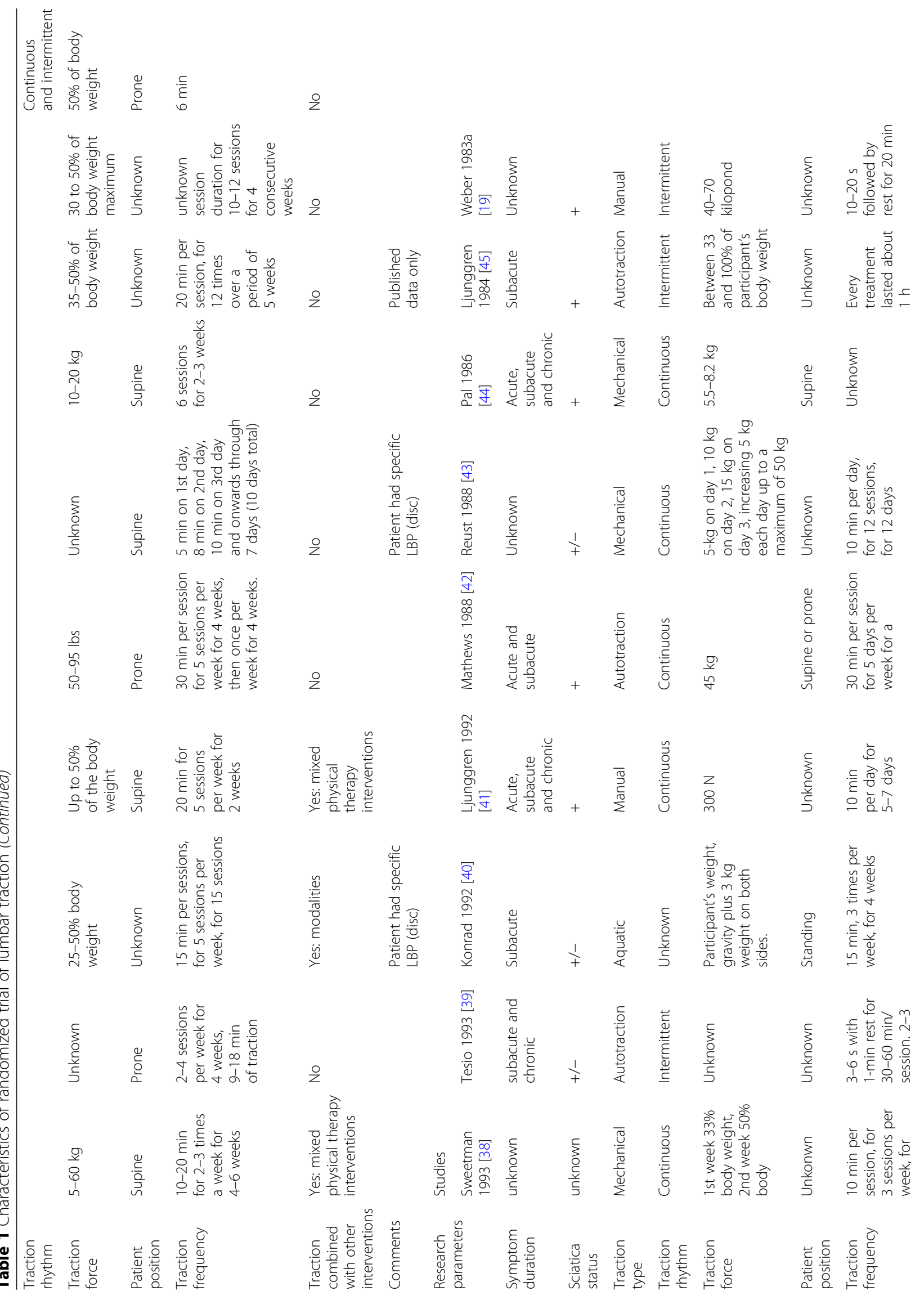




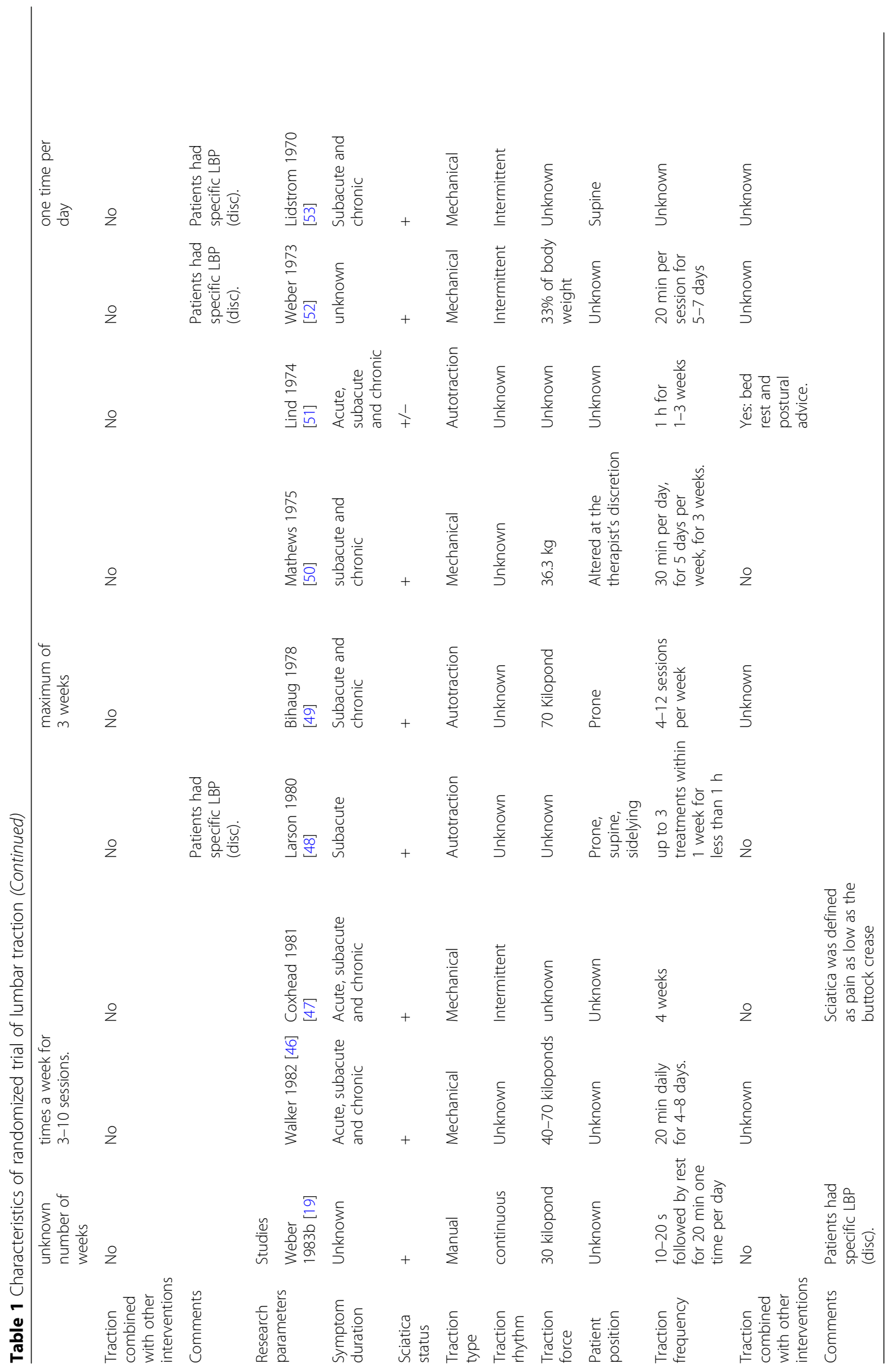




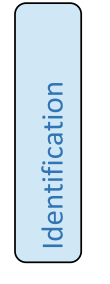

Systematic reviews identified through database
searching
$(n=4)$
RCTs identified through systematic reviews of traction
$(n=32)$

$$
(n=32)
$$
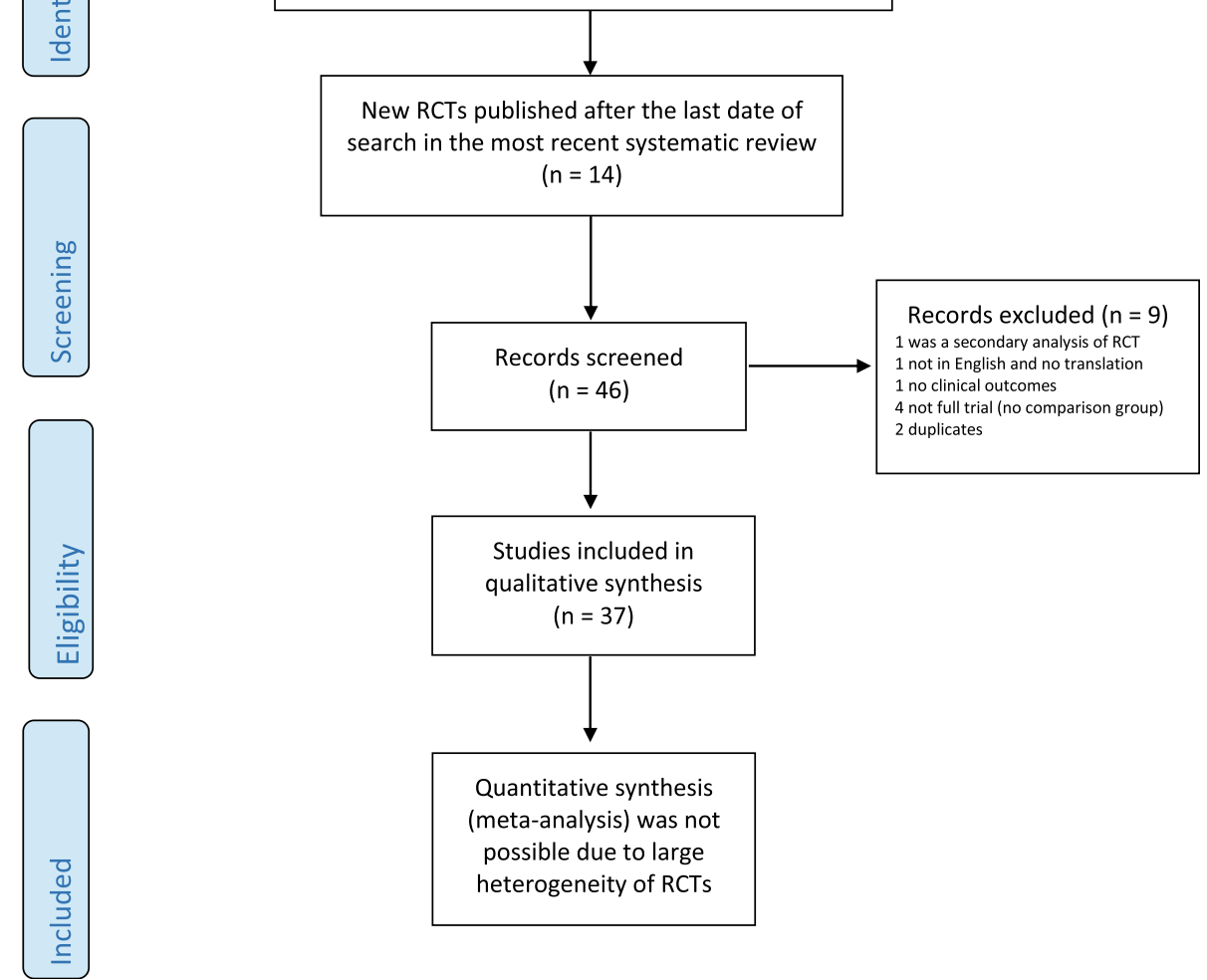

Fig. 1 Flow diagram of data searching, screening and inclusion of traction trials. RCT: Randomized controlled trial

Regarding the onset and duration of symptoms, 54\% of the studies included patients with a mixed acuity of LBP, $16 \%$ included only patients with chronic LBP, $8 \%$ included only subacute cases of LBP, $8 \%$ included only acute cases, and $14 \%$ of the studies did not provide any clear description of symptom acuity. With respect to leg symptoms (e.g. sciatica), 59\% of the RCTs included only patients with sciatica, $24.3 \%$ included a mixture of patients with and without sciatica, $10 \%$ included only patients without sciatica, and the remaining studies did not contain any report of leg symptom or sciatica status. In $24 \%$ of the included studies, the patients had a specific LBP diagnosis such as herniated disc, while the other $76 \%$ of studies did not report any specific pain generator or anatomical cause of the LBP (non-specific LBP).

\section{Discussion}

The results of this systematic review show that there are widespread variations in most of the traction protocols used in the RCTs found in the traction literature. When examining Table 1, each RCT appears to have a distinct combination of traction type and traction parameters applied to different populations of patients with LBP, with or without sciatica. There is no main theme or pattern that emerges about the traction parameters used in the studies included and rated in the previously published systematic reviews. This variability in traction delivery protocols represents a primary gap in the traction literature and might be a key factor that underpins the negative conclusions about traction in the most recent systematic review [5].

\section{Mechanical and non-mechanical traction}

The majority of RCTs that we reviewed used some type of mechanical traction, which involved various devices (e.g. ActiveTrac Table [11], VAX-D decompression [20], SpinaTrac [19], etc.) that used computerized algorithms to produce controlled, intermittent traction forces via motorized pulleys. Although these mechanical devices have the capacity to generate specific forces and rhythms that can be quantified, we found a serious lack of any standardized traction protocol across the RCTs that 
Table 2 Descriptive statistics of traction and patient characteristics

\begin{tabular}{|c|c|}
\hline Traction and patient characteristics & $\begin{array}{l}\text { Descriptive statistics } \\
n(\%)\end{array}$ \\
\hline \multicolumn{2}{|l|}{ Traction types } \\
\hline Mechanical & $22(59.5 \%)$ \\
\hline Manual & $4(10.8 \%)$ \\
\hline Auto-traction & $6(16.2 \%)$ \\
\hline Inversion & $3(8.1 \%)$ \\
\hline Aquatic & $2(5.4 \%)$ \\
\hline \multicolumn{2}{|l|}{ Traction combined with other intervention } \\
\hline Traction alone & $25(67.6 \%)$ \\
\hline Modalities & $1(2.7 \%)$ \\
\hline Directional preference & $2(5.4 \%)$ \\
\hline Mixed with physical therapy interventions & $5(13.5 \%)$ \\
\hline Mixed with unknown intervention & $4(10.8 \%)$ \\
\hline \multicolumn{2}{|l|}{ Traction force } \\
\hline Specific amount of weight (2.3-60 kg) & $14(37.8 \%)$ \\
\hline Percentage of body weight (20-100\%) & $13(35.1 \%)$ \\
\hline Unknown & $10(27 \%)$ \\
\hline \multicolumn{2}{|l|}{ Traction rhythm } \\
\hline Intermittent & $13(35.1 \%)$ \\
\hline Continuous & $14(37.8 \%)$ \\
\hline Mixed & $2(5.4 \%)$ \\
\hline Unknown & $8(21.6 \%)$ \\
\hline \multicolumn{2}{|l|}{ Patient position } \\
\hline Supine & $11(29.7 \%)$ \\
\hline Prone & $6(16.2 \%)$ \\
\hline Standing or vertical & $2(5.4 \%)$ \\
\hline Mixed & $2(5.4 \%)$ \\
\hline Unknown & $16(43.2 \%)$ \\
\hline \multicolumn{2}{|l|}{ Patient symptom duration } \\
\hline Acute & $3(8.1 \%)$ \\
\hline Subacute & $3(8.1 \%)$ \\
\hline Chronic & $6(16.2 \%)$ \\
\hline Mixed & $20(54.1 \%)$ \\
\hline Unknown & $5(13.5 \%)$ \\
\hline \multicolumn{2}{|l|}{ Patient sciatica status } \\
\hline Present sciatica & $22(59.5 \%)$ \\
\hline Absent sciatica & $4(10.8 \%)$ \\
\hline Mixed & $9(24.3 \%)$ \\
\hline Unknown & $2(5.4 \%)$ \\
\hline \multicolumn{2}{|l|}{ Pathology } \\
\hline Specific low back pain pathology & $9(24.3 \%)$ \\
\hline Non-specific low back pain pathology & $28(75.7 \%)$ \\
\hline
\end{tabular}

involved mechanical traction. Instead, the RCTs were found to have great variations in the way the patients were positioned, the duration of traction sessions, the frequency of traction treatment, the amount of traction force applied and the rhythm of traction force. This lack of standardized mechanical traction protocol argues against pooling all of these RCTs under the umbrella term "mechanical traction", and further casts doubt on any conclusions derived from analyses of the pooled studies through meta-analysis.

There were also some studies that utilized non-mechanical traction interventions, such as manual traction, auto-traction, and gravitational traction. All of these interventions present a challenge when attempting to standardize the treatment because the nature of the used traction force cannot be quantified. Manual traction and auto-traction involve traction forces that are dependent on the skill and strength of the clinician and patient respectively. Gravitational (inversion) traction devices and aquatic traction involve traction forces that are dependent on the suspension effect of gravity or water which varies according to the patient's body weight and/ or externally applied weight attachments. These differences in force levels exist among the patients within any one specific trial or across the RCTs. This suggests that the RCTs utilizing non-mechanical traction interventions should not be pooled together, and any conclusions drawn from these inappropriately pooled studies should be considered circumspect.

\section{Determining the traction force}

In the RCTs that involved mechanical traction devices, the traction forces were applied using either a predetermined amount of weight or a percentage of body weight. In many instances, the predetermined amount of weight was not reported (Tables 1 and 2). We could not find any consistent pattern, explanation or scientific rationale that explained the process by which the specific amount of traction force was determined; rather, the process seemed arbitrary across the RCTs.

Compared to using a predetermined amount of weight, using a percentage of body weight would seem to be a better method to individualize, quantify and standardize the traction force. However, the use of a percentage of body weight also varied widely across the RCTs. Despite the belief that a traction force of $25 \%$ of body weight (or above) is reported to create separation between lumbar vertebra $[5,21]$, it remains to be determined what level or range of traction force is optimal and most therapeutic.

\section{Duration and frequency of traction session}

The application time of traction during each treatment session, and the frequency/total number of treatment 
sessions also varied and were distinct in almost every $\mathrm{RCT}$, which renders them difficult to categorize. By examining Table 1, these traction parameters of duration and frequency seem to have been chosen arbitrarily, and therefore we could not determine any consistent traction dosage protocol across the RCTs.

\section{Patients selection for traction}

With respect to patients' characteristics and selection for traction, most of the RCTs included patients with a mixture of symptom duration (i.e. acute, subacute and chronic LBP), with and without sciatica (Table 1). The RCTs did not report any responders and non-responders analyses, which are often performed as a secondary analysis in an attempt to determine if there are any baseline characteristics that might be potential predictors of treatment response. Significant predictor variables can be used to develop clinical prediction rules that can be useful to clinicians for subgrouping patients, and matching treatments with appropriate patient presentations. We only found two such studies; one that developed a preliminary prediction rule for traction and the other that tested this rule within the context of a clinical trial $[11,16]$. This suggests that little attention was given to the homogeneity of LBP population that received lumbar traction within RCTs.

When traction (or any treatment) is applied to all patients without regard to subgroup matching, it is not surprising to find mixed results regarding its clinical effectiveness. This situation reminds us of the discordance between clinicians who practice manual therapy and the literature regarding the effectiveness of spinal manipulation for patients with non-specific LBP [22]. Collectively, spinal manipulation studies have been shown to have only modest treatment effect sizes on LBP when the results of those studies are pooled together in systematic reviews [23]. Meanwhile, rehabilitation providers continue to assert its clinical effectiveness, especially for patients who are matched to clinical prediction rules for manipulation [24]. Not surprisingly, when individual manipulation studies applied the concept of subgrouping and administered manipulation in a matched/unmatched design, the clinical effect sizes were much greater [24, 25]. The same principle of subgrouping has been shown to lead to greater treatment effects in trials that matched specific exercises to patients who exhibited a clear directional preference to flexion or extension movements [26, 27].

There is strong face validity to the concept of using spinal traction as a clinical tool for the treatment of LBP. Spinal traction might work by relieving the stress on a painful joint through increasing intervertebral space, loosening adhesions on facet joints and decreasing mechanical stress on the disc [5]. However, there is simply an evidence gap with respect to a validated clinical prediction rule that could guide the selection of traction for those LBP patients who are more likely to be traction responders.

\section{Future directions for traction}

We suggest that future traction research studies should strive toward standardizing the delivery method of traction for patients with LBP. This could be achieved by focusing more on efficacy trials that explore the clinical effects of different dosage parameters including the traction force level, traction rhythm, traction session duration, and traction treatment frequency. It is possible that traction trials have failed to show a treatment effect simply due to suboptimal dosage. Finding the therapeutic dosage level is key for any treatment to succeed.

Also, future traction research should attempt to provide evidence for subgroup(s) of patients as potential traction responders. This would require some modifications to the research design that focus on baseline characteristics of the patient or the clinical examination. For example, RCTs may be improved by considering the patient's response to a single traction session at first encounter. Patients who show a positive response could be considered to have a "directional preference" to an axial force, and this response could be used as a baseline independent variable in regression models. It would be important to analyze whether the presence of directional preference to an axial force is associated with improved therapeutic effect of traction.

There has been a tendency amongst the RCTs to focus on the inclusion criterion of presence of leg pain (sciatica) and/or signs of nerve root tension, with the assumption that these are important characteristics for a positive response to traction treatment. However, surveys of rehabilitation providers indicate that traction may also be successful with patients who do not present with distal leg symptoms or nerve root compression signs [1, 2]. Future trials should examine if traction force and parameters are different between patients with leg symptoms and patients without leg symptoms.

\section{Conclusions}

RCTs of lumber traction have employed a mixture of traction types, traction parameters and patient populations. The large variability in the delivery of traction intervention provides evidence that the RCTs included in systematic reviews were extremely heterogeneous. This suggests that negative conclusions about the overall clinical effectiveness of lumbar traction should be interpreted with caution. More research about the effectiveness of traction is still necessary, and future trials should consider two important points: (1) discovering optimal dosage and traction parameters to inform the development of standardized traction protocols, and (2) discovering the important baseline variables predictive of successful traction response. By standardizing the traction dosage and parameters, improving patient selection criteria, and response to axial force, more clinically meaningful traction research could be conducted. 


\section{Availability of data and materials}

The datasets used and/or analyzed during the current study are available from the corresponding author on reasonable request.

\section{Authors' contributions}

All authors gave substantial contributions to conception and design, acquisition of data, analysis and interpretation of data; All authors have been involved in drafting the manuscript and revising it critically for important intellectual content; All authors have given final approval of the version to be published. All authors have participated sufficiently in the work to take public responsibility for appropriate portions of the content; and agreed to be accountable for all aspects of the work in ensuring that questions related to the accuracy or integrity of any part of the work are appropriately investigated and resolved.

\section{Ethics approval and consent to participate} Not applicable.

\section{Consent for publication}

Not applicable.

\section{Competing interests}

The authors declare that they have no competing interests.

\section{Publisher's Note}

Springer Nature remains neutral with regard to jurisdictional claims in published maps and institutional affiliations.

\section{Author details}

'Division of Physical Therapy, School of Medicine, West Virginia University, 1 Medical Center Drive, P.O. Box 9226 - Room 8304, Morgantown, WV 26506, USA. ${ }^{2}$ King Fahad Specialist Hosptial, Dammam, Saudi Arabia. ${ }^{3}$ Department of Physical Therapy, School of Applied Medical Sciences, Najran University, King Abdulaziz Rd, PO Box 1988, Najran 61441, Saudi Arabia. ${ }^{4}$ Department of Physical Therapy, School of Health and Rehabilitation Sciences, University of Pittsburgh, Bridgeside Point 1, 100 Technology Drive, Suite 210, Pittsburgh, PA 15219, USA.

\section{Received: 30 October 2017 Accepted: 5 July 2018}

Published online: 17 September 2018

\section{References}

1. Harte AA, Gracey JH, Baxter GD. Current use of lumbar traction in the management of low back pain: results of a survey of physiotherapists in the United Kingdom. Arch Phys Med Rehabil. 2005;86(6):1164-9.

2. Madson TJ, Hollman JH. Lumbar traction for managing low back pain: a survey of physical therapists in the United States. J Orthop Sports Phys Ther. 2015:45(8):586-95.

3. Clarke J, van Tulder M, Blomberg S, de Vet H, van der Heijden G, Bronfort G. Traction for low-back pain with or without sciatica (review). 2005.

4. Clarke J, van Tulder M, Blomberg S, de Vet H, van der Heijden G, Bronfort G. Traction for low back pain with or without sciatica: an updated systematic review within the framework of the Cochrane collaboration. Spine. 2006; 31(14):1591-9.

5. Wegner I, Widyahening IS, van Tulder MW, Blomberg SE, de Vet HC, Brønfort G, et al. Traction for low-back pain with or without sciatica. Cochrane Libr. 2013;

6. Schiotz EH, Cyriax JH. Manipulation past and present: with an extensive bibliography. London: Heinemann Medical; 1975. vi, 222 p. p.

7. van der Heijden GJ, Beurskens AJ, Koes BW, Assendelft WJ, de Vet HC, Bouter LM. The efficacy of traction for back and neck pain: a systematic, blinded review of randomized clinical trial methods. Phys Ther. 1995;75(2):93-104.

8. Alrwaily M, Timko M, Schneider M, Stevans J, Bise C, Hariharan K, et al. Treatment-based classification system for low back pain: revision and update. Phys Ther. 2016;96(7):1057-66.

9. Fritz JM, Cleland JA, Childs JD. Subgrouping patients with low back pain: evolution of a classification approach to physical therapy. J Orthop Sports Phys Ther. 2007;37(6):290-302.
10. Delitto A, Erhard RE, Bowling RW. A treatment-based classification approach to low back syndrome: identifying and staging patients for conservative treatment. Phys Ther. 1995;75(6):470-85. discussion 85-9

11. Fritz JM, Lindsay W, Matheson JW, Brennan GP, Hunter SJ, Moffit SD, et al. Is there a subgroup of patients with low back pain likely to benefit from mechanical traction?: results of a randomized clinical trial and subgrouping analysis. Spine. 2007;32(26):E793-800.

12. Prasad KM, Gregson BA, Hargreaves G, Byrnes T, Winburn P, Mendelow AD. Inversion therapy in patients with pure single level lumbar discogenic disease: a pilot randomized trial. Disabil Rehabil. 2012;34(17):1473-80.

13. Kim J-D, Oh H-W, Lee J-H, Cha J-Y, Ko I-G, Jee Y-S. The effect of inversion traction on pain sensation, lumbar flexibility and trunk muscles strength in patients with chronic low back pain. Isokinet Exerc Sci. 2013;21(3):237-46.

14. Simmerman SM, Sizer PS, Dedrick GS, Apte GG, Brismée J-M. Immediate changes in spinal height and pain after aquatic vertical traction in patients with persistent low back symptoms: a crossover clinical trial. PM\&R. 2011; 3(5):447-57

15. Diab AAM, Moustafa IM. The efficacy of lumbar extension traction for sagittal alignment in mechanical low back pain: a randomized trial. J Back Musculoskelet Rehabil. 2013;26(2):213-20.

16. Thackeray A, Fritz JM, Childs JD, Brennan GP. The effectiveness of mechanical traction among subgroups of patients with low back pain and leg pain: a randomized trial. J Orthop Sports Phys Ther. 2016;46(3):144-54.

17. Isner-Horobeti M-E, Dufour SP, Schaeffer M, Sauleau E, Vautravers P, Lecocq J, et al. High-force versus low-force lumbar traction in acute lumbar sciatica due to disc herniation: a preliminary randomized trial. J Manip Physiol Ther. 2016:39(9):645-54.

18. Moher D, Liberati A, Tetzlaff J, Altman DG. Preferred reporting items for systematic reviews and meta-analyses: the PRISMA statement. J Clin Epidemiol. 2009:62(10):1006-12.

19. Weber $H$, Ljunggren $A$, Walker $L$. Traction therapy in patients with herniated lumbar intervertebral discs. J Oslo City Hosp. 1983;34(7-8):61-70.

20. Sherry E, Kitchener P, Smart R. A prospective randomized controlled study of VAX-D and TENS for the treatment of chronic low back pain. Neurol Res. 2001;23(7):780-4.

21. Beurskens AJ, Henrica C, Köke AJ, Regtop W, van der Heijden GJ, Lindeman E, et al. Efficacy of traction for nonspecific low back pain: 12-week and 6month results of a randomized clinical trial. Spine. 1997;22(23):2756-62.

22. Koes BW, Assendelft WJ, Van der Heijden GJ, Bouter LM. Spinal manipulation for low back pain: an updated systematic review of randomized clinical trials. Spine. 1996;21(24):2860-71.

23. Assendelft WJ, Morton SC, Emily IY, Suttorp MJ, Shekelle PG. Spinal manipulative therapy for low back PainA meta-analysis of effectiveness relative to other therapies. Ann Intern Med. 2003;138(11):871-81.

24. Flynn T, Fritz J, Whitman J, Wainner R, Magel J, Rendeiro D, et al. A clinical prediction rule for classifying patients with low back pain who demonstrate short-term improvement with spinal manipulation. Spine (Phila Pa 1976). 2002;27(24):2835-43

25. Childs JD, Fritz JM, Flynn TW, Irrgang JJ, Johnson KK, Majkowski GR, et al. A clinical prediction rule to identify patients with low back pain most likely to benefit from spinal manipulation: a validation study. Ann Intern Med. 2004; 141(12):920-8

26. Browder DA, Childs JD, Cleland JA, Fritz JM. Effectiveness of an extensionoriented treatment approach in a subgroup of subjects with low back pain: a randomized clinical trial. Phys Ther. 2007;87(12):1608-18.

27. Long A, Donelson R, Fung T. Does it matter which exercise?: a randomized control trial of exercise for low back pain. Spine. 2004;29(23):2593-602.

28. Schimmel JJ, de Kleuver M, Horsting P, Spruit M, Jacobs W, van Limbeek J. No effect of traction in patients with low back pain: a single Centre, single blind, randomized controlled trial of intervertebral differential dynamics therapy ${ }^{\circledast}$. Eur Spine J. 2009;18(12):1843.

29. Unlu Z, Tasci S, Tarhan S, Pabuscu Y, Islak S. Comparison of 3 physical therapy modalities for acute pain in lumbar disc herniation measured by clinical evaluation and magnetic resonance imaging. J Manip Physiol Ther. 2008;31(3):191-8.

30. Harte AA, Baxter GD, Gracey JH. The effectiveness of motorised lumbar traction in the management of LBP with lumbo sacral nerve root involvement: a feasibility study. BMC Musculoskelet Disord. 2007:8(1):118.

31. Gudavalli MR, Cambron JA, McGregor M, Jedlicka J, Keenum M, Ghanayem AJ, et al. A randomized clinical trial and subgroup analysis to compare flexion-distraction with active exercise for chronic low back pain. Eur Spine J. 2006;15(7):1070-82. 
32. Ozturk B, Gunduz OH, Ozoran K, Bostanoglu S. Effect of continuous lumba traction on the size of herniated disc material in lumbar disc herniation. Rheumatol Int. 2006;26(7):622-6.

33. Borman $\mathrm{P}$, Keskin $\mathrm{D}$, Bodur $\mathrm{H}$. The efficacy of lumbar traction in the management of patients with low back pain. Rheumatol Int. 2003;23(2):82-6.

34. Güevenol K, Tüzün Ç, Peker Ö, Göktay Y. A comparison of inverted spinal traction and conventional traction in the treatment of lumbar disc herniations. Physiother Theory Pract. 2000;16(3):151-60.

35. Werners R, Pynsent PB, Bulstrode CJ. Randomized trial comparing interferential therapy with motorized lumbar traction and massage in the management of low back pain in a primary care setting. Spine. 1999;24(15):1579.

36. Van der Heijden G, Beurskens A, Dirx M, Bouter L, Lindeman E. Efficacy of lumbar traction: a randomised clinical trial. Physiotherapy. 1995;81(1):29-35.

37. Letchuman $\mathrm{R}$, Deusinger $\mathrm{RH}$. Comparison of sacrospinalis myoelectric activity and pain levels in patients undergoing static and intermittent lumbar traction. Spine. 1993:18(10):1361-5.

38. Sweetman B, Heinrich I, Anderson J. A randomized controlled trial of exercises, short wave diathermy and traction for low back pain, with evidence of diagnosis related response to treatment. J Orthop Rheumatol. 1993;6:159.

39. Tesio L, Merlo A. Autotraction versus passive traction: an open controlled study. Arch Phys Med Rehabil. 1993;74

40. Konrad K, Tatrai T, Hunka A, Vereckei E, Korondi I. Controlled trial of balneotherapy in treatment of low back pain. Ann Rheum Dis. 1992; 51(6):820-2.

41. Ljunggren $A E$, Walker $L$, Weber $H$, Amundsen $T$. Manual traction versus isometric exercises in patients with herniated intervertebral lumbar discs. Physiother Theory Pract. 1992;8(4):207-13.

42. Mathews J, Mills S, Jenkins V, Grimes S, Morkel M, Mathews W, et al. Back pain and sciatica: controlled trials of manipulation, traction, sclerosant and epidural injections. Rheumatology. 1987;26(6):416-23.

43. Reust $P$, Chantraine A, Vischer T. Treatment of lumbar sciatica with or without neurological deficit using mechanical traction. A double-blind study. Schweiz Med Wochenschr. 1988;118(8):271.

44. Pal B, Mangion P, Hossain M, Diffey B. A controlled trial of continuous lumbar traction in the treatment of back pain and sciatica. Rheumatology. 1986;25(2):181-3

45. Ljunggren $\mathrm{A}$, Weber $\mathrm{H}$, Larsen $\mathrm{S}$. Autotraction versus manual traction in patients with prolapsed lumbar intervertebral discs. Scand J Rehabil Med. 1983;16(3):117-24

46. Walker L, Svenkerud T, Weber H. Traksjonsbehandling ved lumbago-ischias: en kontrollert undersolske med Spina-trac. Fysioterapeuten. 1982;49:161-3.

47. Coxhead C, Meade T, Inskip H, North W, Troup J. Multicentre trial of physiotherapy in the management of sciatic symptoms. Lancet. 1981; 317(8229):1065-8.

48. Larsson U, Chöler U, Lidström A, Lind G, Nachemson A, Nilsson B, et al. Auto-traction for treatment of lumbago-sciatica: a multicentre controlled investigation. Acta Orthop Scand. 1980;51(1-6):791-8.

49. Bihaug O. Autotraksjon for ischialgpasienter: en kontrollert sammenlikning mellom effekten av Auto-traksjon-B og isometriske ovelser ad modum Hume endall og enkins. Fysioterapeuten. 1978;45:377-9.

50. Mathews JA, Hickling J. Lumbar traction: a double-blind controlled study for sciatica. Rheumatology. 1975;14(4):222-5.

51. Lind GA. Auto-traction: treatment of low back pain and sciatica: an electromyographic, radiographic and clinical study: publisher not identified; 1974.

52. Weber $\mathrm{H}$. Traction therapy in sciatica due to disc prolapse (does traction treatment have any positive effect on patients suffering from sciatica caused by disc prolapse?). J Oslo City Hosp. 1973:23(10):167.

53. Lidström A, Zachrisson M. Physical therapy on low back pain and sciatica. An attempt at evaluation Scandinavian journal of rehabilitation medicine. 1970;2(1):37-42.

\section{Ready to submit your research? Choose BMC and benefit from:}

- fast, convenient online submission

- thorough peer review by experienced researchers in your field

- rapid publication on acceptance

- support for research data, including large and complex data types

- gold Open Access which fosters wider collaboration and increased citations

- maximum visibility for your research: over $100 \mathrm{M}$ website views per year

At BMC, research is always in progress.

Learn more biomedcentral.com/submissions 